\title{
Elevation of Unsaturated Lipid Species is Accompanied by the Upregulation of Stearoyl-CoA Desaturase in Ovarian Clear Cell Carcinoma
}

\section{Sartaj Ahmad Mir ( $\sim$ bchsam@nus.edu.sg )}

National University of Singapore https://orcid.org/0000-0001-6032-0936

Soon Boon Justin Wong

National University Hospital, Singapore

Kothandaraman Narasimhan

Singapore Institute for Clinical Sciences

Chua Wee Ling Esther

National University of Singapore

Shanshan Ji

National University of Singapore

\section{Bo Burla}

National University of Singapore

Markus R. Wenk

National University of Singapore

David S.P. Tan

National University Hospital

Anne K. Bendt

National University of Singapore

\section{Research}

Keywords: mass spectrometry, ovarian cancer, lipid profiling, unsaturated fatty acids, desaturation, archival, formalin-fixed

Posted Date: February 18th, 2021

DOI: https://doi.org/10.21203/rs.3.rs-207019/v1

License: (c) (1) This work is licensed under a Creative Commons Attribution 4.0 International License.

Read Full License 


\section{Abstract}

\section{Background}

Cancer metabolism is associated with enhanced lipogenesis required for rapid growth and proliferation. However, the magnitude of dysregulation of diverse lipid species mostly remain to be characterized, particularly in understudied cancers such as ovarian clear cell carcinoma (OCCC). Further, formalin-fixed paraffin-embedded (FFPE) specimen represent valuable and readily available resources for laboratory investigations but are still underutilized for lipidomics studies. Here we implemented an integrated workflow to investigate lipidomic alterations in OCCC, utilizing FFPE specimen.

\section{Methods}

Lipid extraction of FFPE specimen was carried out by using 1-butanol and methanol in 1:1 (v/v), followed by targeted LC-MS/MS to identify the lipidomic changes in FFPE specimen from OCCC $(n=14)$ as compared to uninvolved contralateral ovarian tissue $(n=14)$. Immunohistochemistry $(\mathrm{IHC})$ assays were implemented to evaluate the expression levels of stearoyl-CoA desaturase (SCD), the rate-limiting enzyme for the synthesis of monounsaturated fatty acids, in OCCC and control tissue specimen.

\section{Results}

We quantified 340 lipid species representing 29 lipid classes. We observed differential regulation of unsaturated lipid species belonging to several glycerophospholipid classes including ether-linked phospholipids, and trihexosylceramide species. We confirmed upregulation of SCD in OCCC by IHC assays.

\section{Conclusions}

By using lipidomic and $\mathrm{IHC}$ analysis of archival tissue samples, we were able to provide novel insights into the molecular alterations in OCCC. We show the feasibility of using FFPE specimen to carry out detailed lipidomic analysis of archival cancer samples.

\section{Full Text}

Due to technical limitations, full-text HTML conversion of this manuscript could not be completed. However, the latest manuscript can be downloaded and accessed as a PDF.

\section{Figures}


Figure 1

(A)

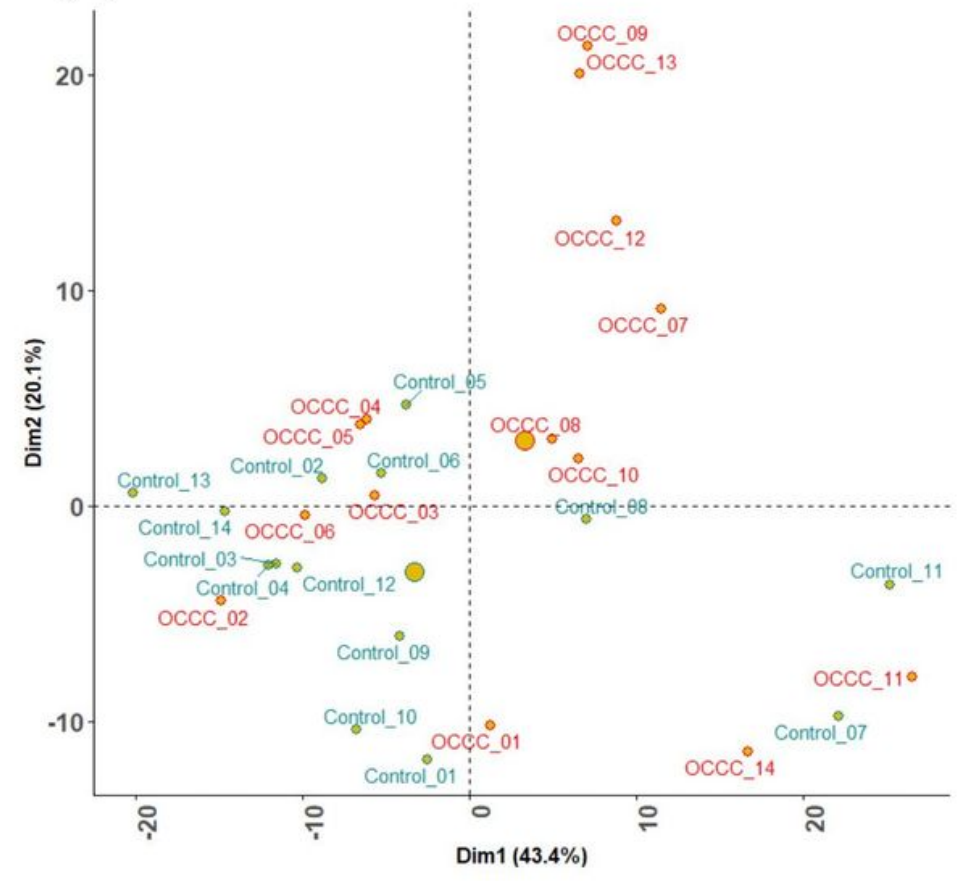

(B)

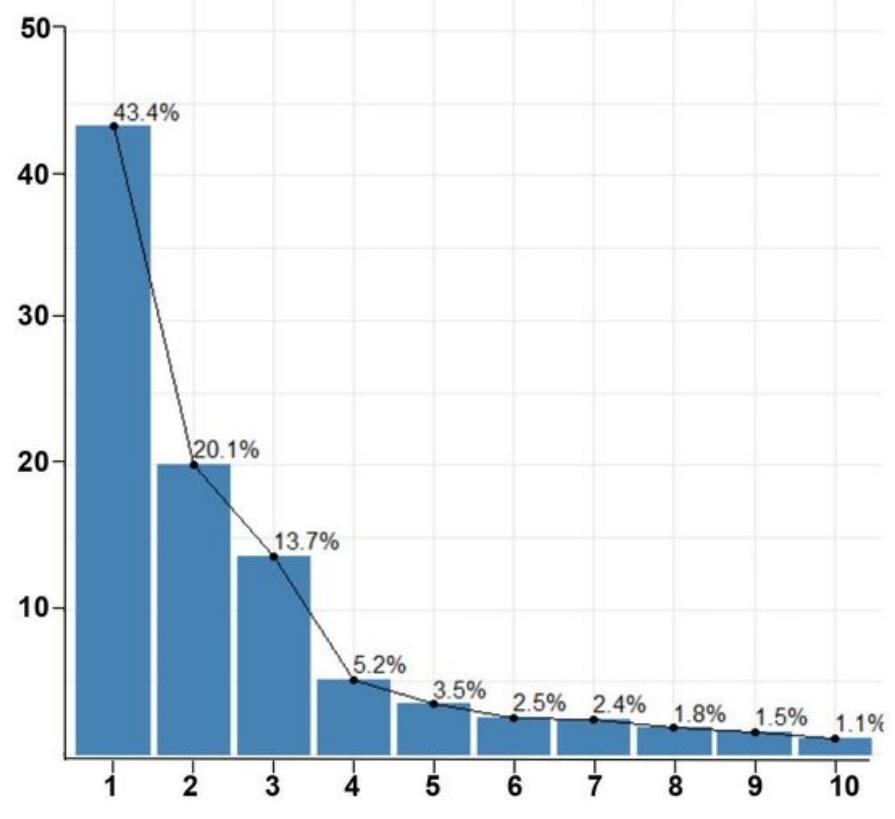

Figure 1

(A) PCA plot based on the lipidomics data of $n=14$ OCCC cases and $n=14$ paired control tissues. $43.4 \%$ variance was explained by PC1 (B) scree plot showing the contribution of top 10 principal components 


\section{Figure 2}

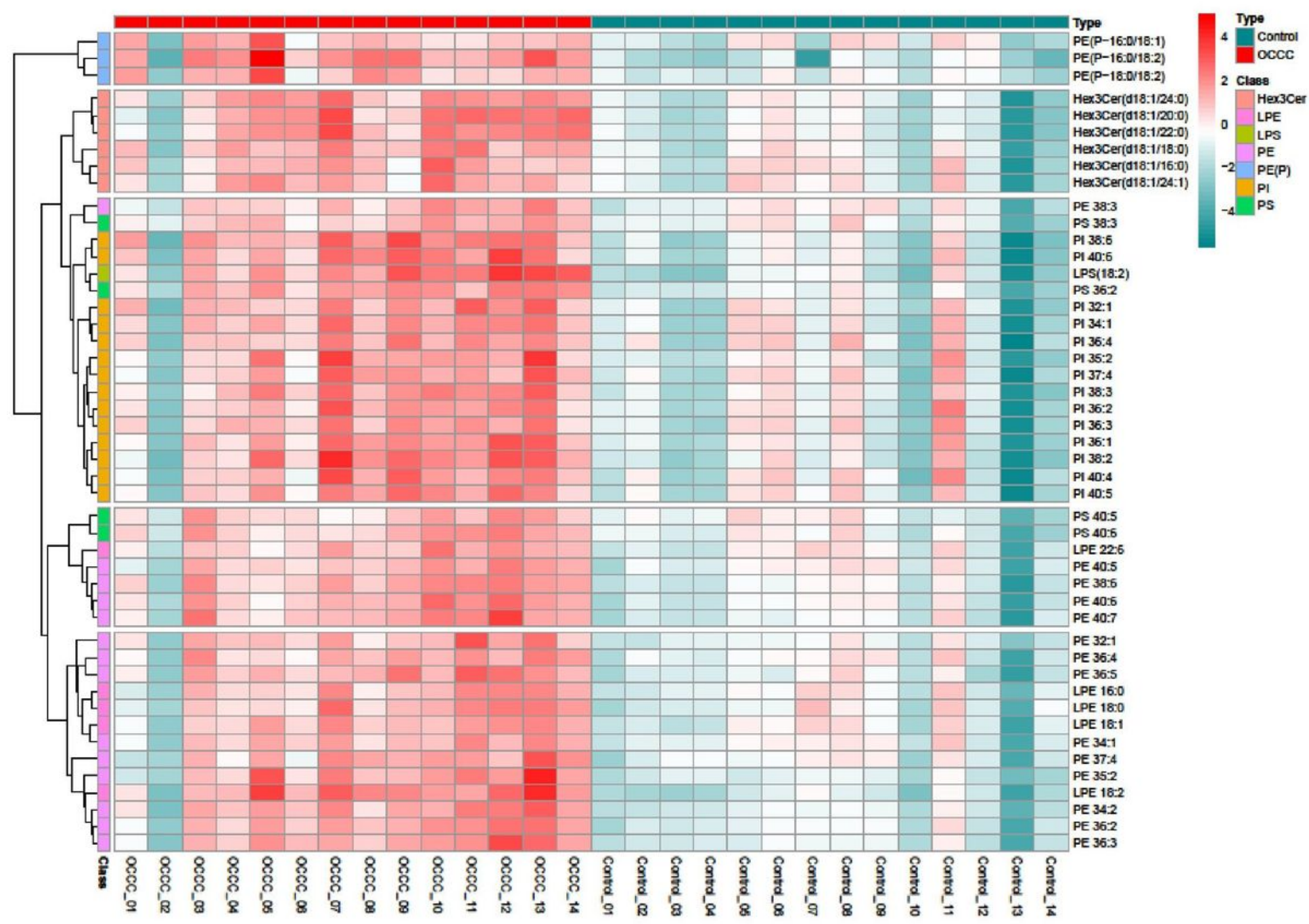

Figure 2

Heatmap with clustering analysis derived from the average log abundance of differentially regulated lipid species. Clustering analysis showed five main clusters based on correlation between lipid species. 
Figure 3
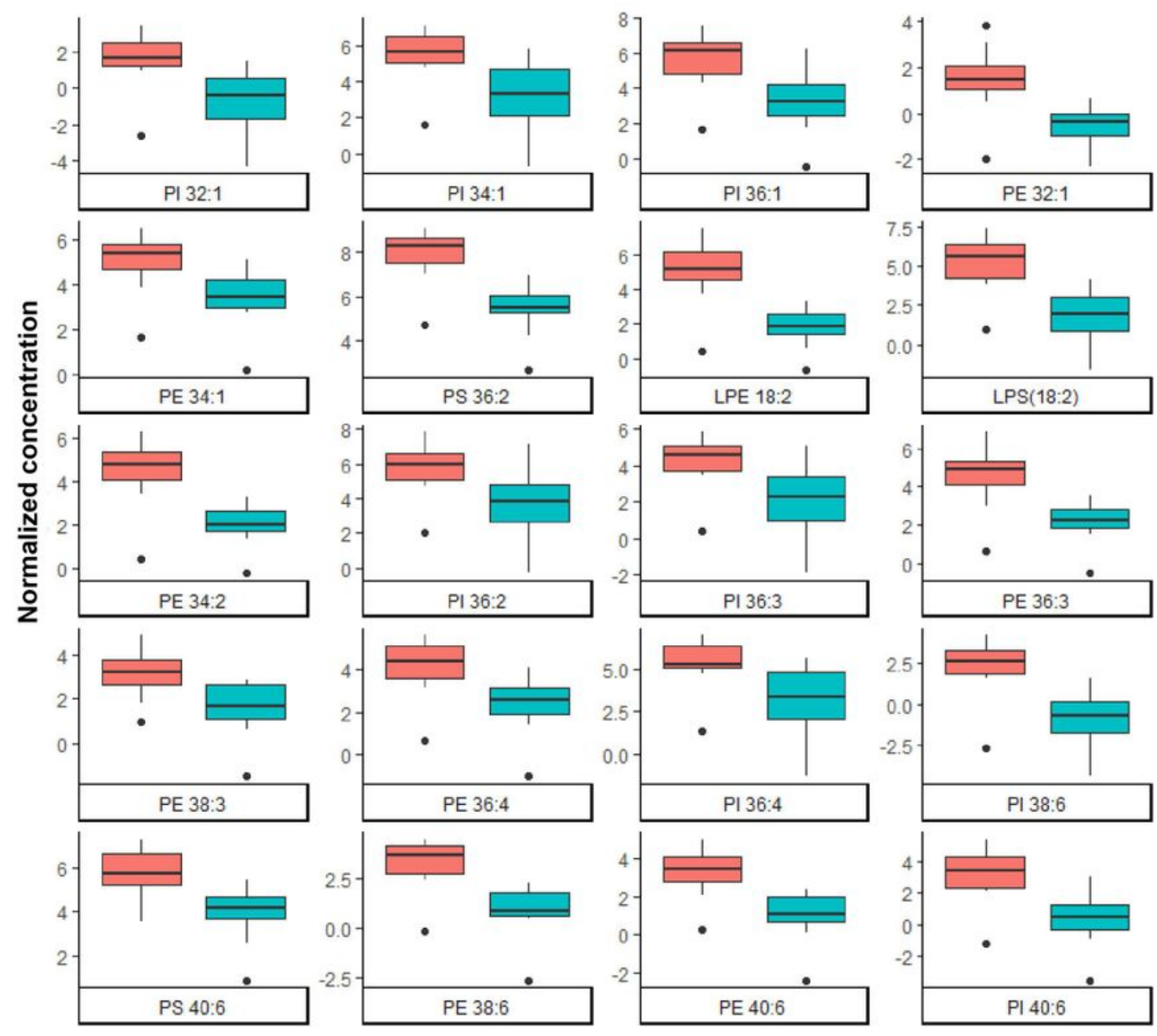

早 OCCC 早 Control

\section{Figure 3}

Representative examples of differentially regulated glycerophospholipid species in OCCC with one or more double bonds. 
Figure 4
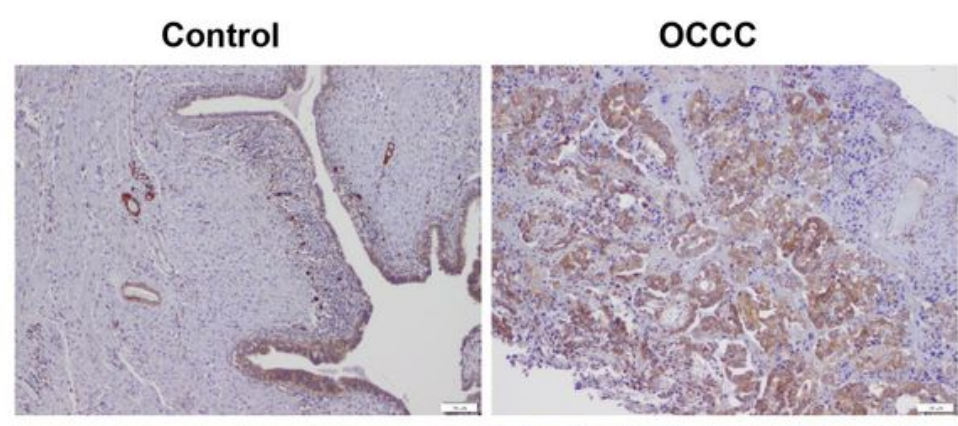

$H \& E$
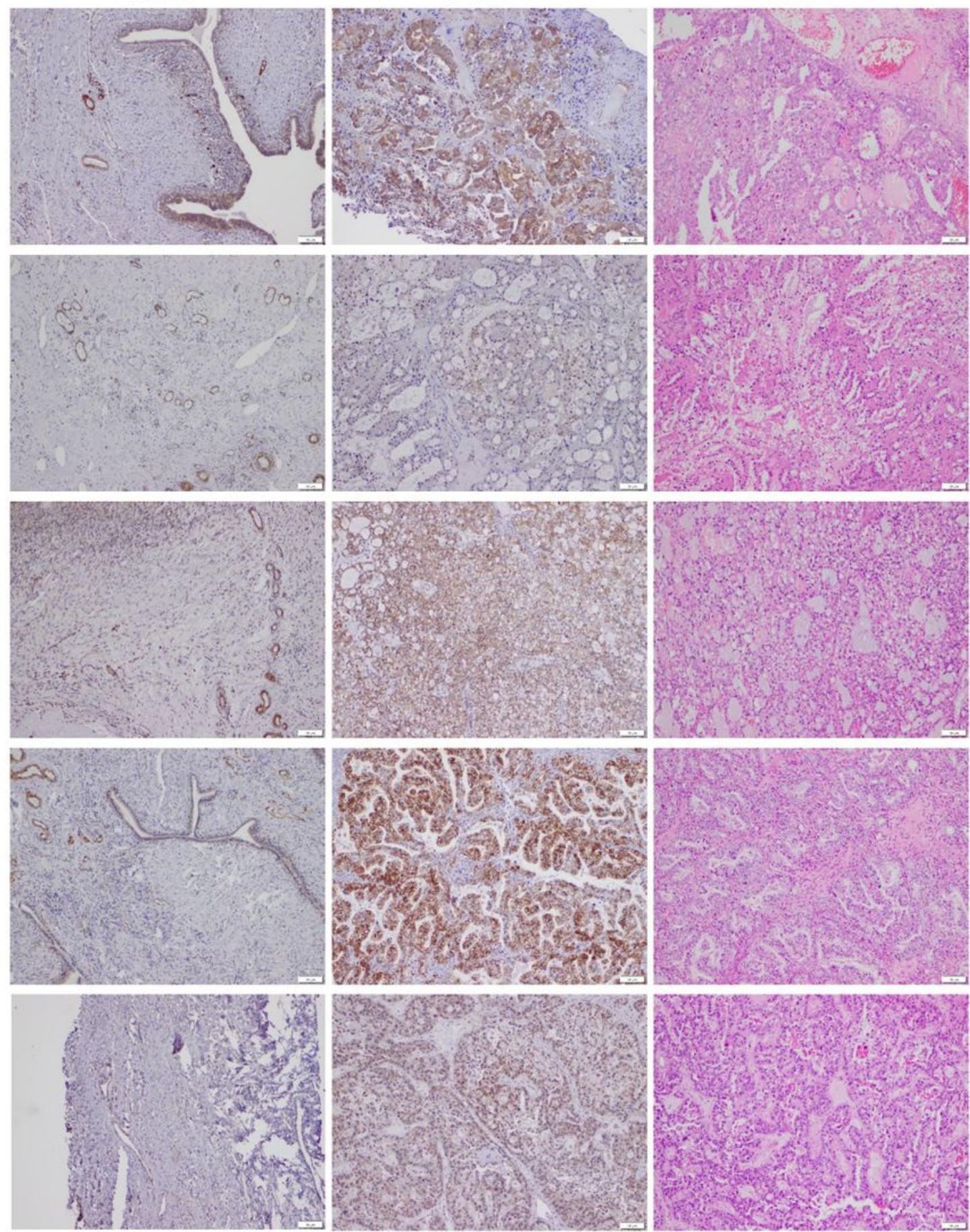

Figure 4

Immunohistochemical analysis of SCD in five OCCC and paired control FFPE sections with corresponding H\&E for each cancer specimen.

\section{Supplementary Files}


This is a list of supplementary files associated with this preprint. Click to download.

- MirSupplementaryFlgures.pdf

- SupplementarylnformationS1S2.xlsx 